\title{
On irreducible, infinite, nonaffine Coxeter groups
}

\author{
by
}

\author{
Dongwen Qi (Columbus, OH)
}

\begin{abstract}
The following results are proved: The center of any finite index subgroup of an irreducible, infinite, nonaffine Coxeter group is trivial; Any finite index subgroup of an irreducible, infinite, nonaffine Coxeter group cannot be expressed as a product of two nontrivial subgroups. These two theorems imply a unique decomposition theorem for a class of Coxeter groups. We also prove that the orbit of each element other than the identity under the conjugation action in an irreducible, infinite, nonaffine Coxeter group is an infinite set. This implies that an irreducible, infinite Coxeter group is affine if and only if it contains an abelian subgroup of finite index.
\end{abstract}

1. Introduction. A Coxeter system $(W, S)$ is a group $W$ and a set $S$ of generators such that $W$ has a presentation of the form

$$
W=\left\langle S \mid(s t)^{m_{s t}}=1, s, t \in S\right\rangle,
$$

where $m_{s t}=m_{t s}$ is a positive integer or $\infty$, and $m_{s t}=1$ if and only if $s=t$. (A relation $(s t)^{\infty}=1$ is interpreted as vacuous.) $W$ is called a Coxeter group. The cardinality $|S|$ of $S$ is called the rank of $W$. We are mainly interested in Coxeter groups of finite rank. So, we assume $|S|$ is finite in this paper.

For a Coxeter system $(W, S)$, its Coxeter graph is a graph with vertex set $S$, and with two vertices $s \neq t$ joined by an edge whenever $m_{s t} \geq 3$. If $m_{s t} \geq 4$, the corresponding edge is labeled by $m_{s t}$. We say that a Coxeter system $(W, S)$ is irreducible if its Coxeter graph is connected.

Associated to a Coxeter group $(W, S)$, there is a symmetric bilinear form on a real vector space $V$, having a basis $\left\{\alpha_{s} \mid s \in S\right\}$ in one-to-one correspondence with $S$. The bilinear form (, ) is defined by setting

$$
\left(\alpha_{s}, \alpha_{t}\right)=-\cos \frac{\pi}{m_{s t}} .
$$

The value on the right-hand side is interpreted to be -1 when $m_{s t}=\infty$.

2000 Mathematics Subject Classification: Primary 20F55; Secondary 20F65, 57M07, $53 \mathrm{C} 23$.

Key words and phrases: irreducible Coxeter groups, parabolic subgroup, essential element, $\mathrm{CAT}(0)$ space, flat torus theorem, solvable subgroup theorem. 
A well known fact is that a Coxeter group $W$ is finite if and only if its bilinear form is positive definite. It is stated in [2, p. 137] that an irreducible, infinite Coxeter group has a trivial center, and a proof using the canonical representations of a Coxeter group, developed by J. Tits (see [2], [16]), is suggested.

If the bilinear form of an irreducible Coxeter system $(W, S)$ is positive semi-definite but not positive definite, then $W=\mathbb{Z}^{n} \rtimes W_{0}$, where $W_{0}$ is a finite Coxeter group and $n=|S|-1$. We call $W$ an irreducible, infinite, affine Coxeter group in this situation. A natural and interesting question, which was proposed to the author by M. Davis and T. Januszkiewicz, is to determine if the center of a finite index subgroup of an irreducible, infinite, nonaffine Coxeter group is trivial. By "nonaffine" we mean that its bilinear form is neither positive definite nor positive semi-definite. The answer is yes.

THEOREM 1.1. The center of any finite index subgroup of an irreducible, infinite, nonaffine Coxeter group is trivial.

The solution of this question was inspired by a preprint of L. Paris (version 1 of [19]). In his paper, by studying the essential elements (which will be defined in Section 3) of a Coxeter group, Paris obtained several interesting results on irreducible Coxeter groups. One of them is that any irreducible, infinite Coxeter group cannot be written as a product of two nontrivial subgroups. This paper also brought to the author's attention D. Krammer's thesis [17].

The idea of studying essential elements (Krammer [17], Paris [19]) is important in the proof of Theorem 1.1. In addition, we make use of some arguments similar to those in the proofs of the Flat Torus Theorem and the Solvable Subgroup Theorem of CAT(0) spaces. For a detailed description of CAT(0) spaces, the reader is referred to [3]. We will explain briefly in Section 2 a geometric construction associated to a Coxeter system $(W, S)$ (see [6], [7]) which yields a PE cell complex $\Sigma=\Sigma(W, S)$ (here PE stands for "piecewise Euclidean"), now commonly called the Davis complex. It is proved by G. Moussong [18] that $\Sigma$ is a CAT(0) space and $W$ acts properly and cocompactly on $\Sigma$ by isometries.

The proof of Theorem 1.1 also relies on the general theory of the root system of a Coxeter group (see Bourbaki [2], Deodhar [11] and Krammer [17]). Deodhar [12] and M. Dyer [13] independently proved a theorem which says that any subgroup generated by a collection of reflections in a Coxeter group is a Coxeter group itself. This theorem also plays an important role in our proof.

Using some arguments similar to the proof of Theorem 1.1, we obtain the following. 
TheOREm 1.2. Any finite index subgroup of an irreducible, infinite, nonaffine Coxeter group cannot be expressed as a product of two nontrivial subgroups.

After proving these, the author discovered that in a revised version (version 2) of [19], Paris extended his discussions to include the conclusions of Theorem 1.1 and 1.2 using purely algebraic arguments. It appears that we both realized the necessity of use of the reflection subgroup theorem obtained by Deodhar and Dyer to achieve this aim.

A recent work of de Cornulier and de la Harpe [5] provides a different proof of Theorem 1.2, where they mention that Theorem 1.1 can be obtained from a result in Benoist and de la Harpe [1].

Based on Theorems 1.1 and 1.2, we obtain the following result.

THEOREM 1.3. If a group $G$ is a direct product of $n$ irreducible, infinite, nonaffine Coxeter groups, then any finite index subgroup $H$ of $G$ has a trivial center, and $H$ can be expressed uniquely as a direct product of $m$ nontrivial subgroups of $H$ (up to the rearrangement of factors), where each factor cannot be further decomposed and $1 \leq m \leq n$.

There are examples, which will be explained in Section 4, showing that the situation $m<n$ can happen.

Using some arguments of the proof of Theorem 1.1, we obtain another result, which has an implication for the group ring $R[W]$, where $W$ is an irreducible, infinite, nonaffine Coxeter group and $R$ is a commutative ring with identity. Here $R[W]$ is the free $R$-module generated by the elements of $W$. Any element of $R[W]$ is of the form $\sum_{w \in W} a(w) w$, where $a(w) \in R$ and $a(w)=0$ for almost all $w$. The multiplication in $W$ extends uniquely to an $R$-bilinear product $R[W] \times R[W] \rightarrow R[W]$. This makes $R[W]$ a ring. First, we have the following.

THEOREM 1.4. Given an irreducible, infinite, nonaffine Coxeter group $(W, S)$ and any $w \neq 1$ in $W$, the cardinality of the set $\left\{g w g^{-1} \mid g \in W\right\}$ is infinite.

As a comparison, recall that an irreducible, infinite, affine Coxeter group $W$ has a decomposition $\mathbb{Z}^{n} \rtimes W_{0}$, where $W_{0}$ is a finite Coxeter group. In this situation, the cardinality of the set $\left\{g w g^{-1} \mid g \in W\right\}$ is finite for $w \in \mathbb{Z}^{n}$. In summary, Theorems 1.1, 1.2 and 1.4 illustrate the group-theoretic differences between irreducible, infinite, nonaffine Coxeter groups and irreducible, infinite, affine Coxeter groups, even though the classification between "affine" and "nonaffine" is based on generators and relations and the associated bilinear form. Indeed, the following conclusion follows easily from Theorem 1.4. A different proof of this statement can be found in [10, p. 15]. 
Corollary 1.5. An irreducible, infinite Coxeter group $W$ is affine if and only if it contains an abelian subgroup of finite index.

Proof. The "only if" part is obvious. For the "if" part, suppose that $A$ is a finite index abelian subgroup of the irreducible, infinite Coxeter group $W$. Since the number of distinct left cosets of $A$ in $W$ is the same as the number of distinct right cosets of $A$ in $W$, we assume that $W=\bigcup_{i=1}^{n} w_{i} A$, where $n$ is a positive integer, and $\left\{w_{1}, \ldots, w_{n}\right\}$ is a designated finite subset of $W$. Given $a \neq 1 \in A$, we have $\left\{w a w^{-1} \mid w \in W\right\}=\left\{w_{i} a w_{i}^{-1} \mid i=1, \ldots, n\right\}$. By Theorem 1.4, $W$ cannot be nonaffine. Hence, $W$ is affine.

The corollary for the group ring $R[W]$ is as follows; the proof is left to the reader.

Corollary 1.6. Let $W$ be an irreducible, infinite, nonaffine Coxeter group and $R$ be a commutative ring with identity. The center of $R[W]$ is $R$.

The paper is organized as follows. In Section 2, we state some basic facts about the combinatorial theory of a Coxeter group. Using these, we give another proof of the statement that an irreducible, infinite Coxeter group has a trivial center. This proof does not use the canonical representations of Coxeter groups and is of purely combinatorial nature. Then we describe briefly the construction of the Davis complex of a Coxeter group. The root system of a Coxeter group is introduced in Section 3, where some key results from Krammer's thesis are stated. These are important in our discussions. The proofs of the above theorems are given in Section 4 .

2. Basic combinatorial theory of Coxeter groups. A Coxeter group may be characterized by some combinatorial conditions, which are stated below. For now, let $W$ be a group generated by a subset $S$ of involutions (elements of order 2). The length $l(w)$ or $l_{S}(w)$ of an element $w \in W$, with respect to $S$, is the smallest number $d$ such that $w=s_{1} \cdots s_{d}$, with all $s_{i} \in S$. This expression for $w$ is called a reduced decomposition of $w$ if $d=l(w)$.

Consider the following conditions.

(D) Deletion Condition. If $w=s_{1} \cdots s_{d}$ with $d>l(w)$, then there are indices $i<j$ such that $w=s_{1} \cdots \widehat{s}_{i} \cdots \widehat{s}_{j} \cdots s_{d}$, where the hats indicate deleted letters.

(E) Exchange Condition. Given $w \in W, s \in S$, and any reduced decomposition $w=s_{1} \cdots s_{d}$ of $w$, either $l(s w)=d+1$ or else there is an index $i$ such that $w=s s_{1} \cdots \widehat{s}_{i} \cdots s_{d}$.

(F) Folding Condition. Given $w \in W$ and $s, t \in S$ such that $l(s w)=$ $l(w)+1$ and $l(w t)=l(w)+1$, either $l(s w t)=l(w)+2$ or else $s w=w t$.

The proof of the following theorem can be found in [2], [4] or [9]. 
TheOREM 2.1. A group $W$ generated by a set $S$ of involutions gives a Coxeter system $(W, S)$ if and only if $W$ satisfies any one of the conditions $(\mathrm{D}),(\mathrm{E})$ and $(\mathrm{F})$, with the length function $l(w)=l_{S}(w)$ defined as above.

Given a Coxeter system $(W, S)$, for each subset $T$ of $S$, let $W_{T}$ be the subgroup generated by $T$. Call it a special subgroup of $W$. Then any element $w \in W$ can be expressed as $w=w_{0} a$ where $a \in W_{T}$ and $w_{0}$ is the shortest element in the left coset $w W_{T}$. This $w_{0}$ is characterized by the property $l\left(w_{0} t\right)=l\left(w_{0}\right)+1$ for any $t \in T$ and it is unique in $w W_{T}$. We say $w_{0}$ is $(\emptyset, T)$-reduced in this situation. It is clear this type of decomposition for $w$ is unique and $w_{0}$ satisfies $l\left(w_{0} b\right)=l\left(w_{0}\right)+l(b)$ for any $b \in W_{T}$. A similar discussion for right cosets gives a "right-hand version" of the decomposition and the definition of $(T, \emptyset)$-reduced elements.

For $w \in W$, define a subset $\operatorname{In}(w)$ of $S$ by

$$
\operatorname{In}(w)=\{s \in S \mid l(w s)=l(w)-1\},
$$

and put

$$
\operatorname{Out}(w)=S-\operatorname{In}(w) .
$$

We collect some basic facts about finite special subgroups of a Coxeter group.

Lemma 2.2. Suppose $W_{T}$ is a finite subgroup, where $T \subset S$. Then there is a unique element $w_{T}$ in $W_{T}$ of longest length. Moreover:

(1) $w_{T}$ is an involution.

(2) For any $x \in W_{T}, x=w_{T}$ if and only if $\operatorname{In}(x)=T$.

(3) For any $x \in W_{T}, l\left(w_{T} x\right)=l\left(w_{T}\right)-l(x)$.

This lemma is taken from exercises in Chapter 4 of [2]. Its proof, as well as that of the following two lemmas, can be found in Chapter 3 of Davis [9]. For the readers' convenience, we include the proof of Lemma 2.4.

Lemma $2.3([8])$. For any $x \in W, W_{\operatorname{In}(x)}$ is a finite subgroup.

Lemma $2.4([8])$. If $W_{T}$ is a finite subgroup of $W$ and $w_{T}$ is the longest element in $W_{T}$, then for $s \in S-T, s w_{T}=w_{T} s$ if and only if $m_{s t}=2$ for all $t \in T$.

Proof. If $m_{s t}=2$, then $s$ and $t$ commute. Hence, if $m_{s t}=2$ for all $t \in T$, then $s$ and $w_{T}$ commute.

Conversely, suppose $s$ and $w_{T}$ commute, where $s \notin T$. Then $l\left(w_{T} s\right)=$ $l\left(w_{T}\right)+1$, so $s \in \operatorname{In}\left(w_{T} s\right)$. Since $w_{T} s=s w_{T}$, we see that $T \subset \operatorname{In}\left(w_{T} s\right)$. Therefore, $\operatorname{In}\left(w_{T} s\right)=T \cup\{s\}, w_{T \cup\{s\}}=w_{T} s$. We want to show that $m_{s t}=2$ for all $t \in T$. Suppose, to the contrary, that $m_{s t}>2$ for some $t \in T$. Then $l(s t s)=3, l\left(\left(w_{T} s\right)(s t s)\right)=l\left(w_{T}\right)+1-3=l\left(w_{T}\right)-2$ by Lemma 2.2. On the other hand, $l\left(\left(w_{T} s\right)(s t s)\right)=l\left(w_{T} t s\right)=l\left(w_{T} t\right)+1=l\left(w_{T}\right)$, a contradiction. Hence, the conclusion of Lemma 2.4 holds. 
Now we are ready to prove the following statement.

Proposition 2.5. The center of an irreducible, infinite Coxeter group $(W, S)$ is trivial.

Proof. If $w \neq 1$ is in the center of $W$, then $w s=s w$ for any $s \in S$. Put $S_{1}=\operatorname{In}(w)$ and $S_{2}=\operatorname{Out}(w)$. Then $S_{1} \neq \emptyset$. Write $w=w_{0} a$ with $a \in W_{S_{1}}$ and $w_{0}$ being $\left(\emptyset, S_{1}\right)$-reduced. Notice that $l(w)-1=l(w s)=l\left(w_{0} a s\right)$ for any $s \in S_{1}$; it follows that $l(a s)=l(a)-1$ for all $s \in S_{1}$. By Lemmas 2.2 and 2.3, $a$ is the (unique) longest element in the finite subgroup $W_{S_{1}}$, and $a^{2}=1$.

Now, consider the "right-hand version" of the above-mentioned decomposition of $w$. Since $w$ is in the center of $W$, we have $w=a w_{1}$, where $w_{1}$ is $\left(S_{1}, \emptyset\right)$-reduced, $a$ is the longest element in $W_{S_{1}}$. Hence, $w_{0}=w a=a w=w_{1}$, $w=a w_{0}=w_{0} a$.

Notice that $l(w t)=l(w)+1$ for any $t \in S_{2}$, hence $l\left(w_{0} t\right)=l\left(w_{0}\right)+1$, since otherwise we would have $l(w t) \leq l(a)+l\left(w_{0} t\right) \leq l(w)-1$, contradicting the definition of $S_{2}$. Therefore, $w_{0}$ is $\left(\emptyset, S_{2}\right)$-reduced, and hence $(\emptyset, S)$-reduced. This implies that $w_{0}=1$ and $w=a$, i.e., $w=w_{S_{1}}$. So, $w_{S_{1}}$ commutes with every element in $S_{2}=S-S_{1}$. Now, Lemma 2.4 implies that $m_{s t}=2$ for any $s \in S_{1}, t \in S_{2}$. The irreducibility of $W$ implies $S_{2}=\emptyset$ and hence $W$ is a finite Coxeter group, a contradiction. This finishes the proof of Proposition 2.5.

To prove the theorems stated in the introduction, we need the fact that a Coxeter group acts properly and cocompactly on a CAT(0) space. Here we give a brief description of the Davis complex. For a more complete account, the reader is referred to [6]-[8].

Let $(W, S)$ be a Coxeter system. We define a poset, denoted $\mathrm{S}^{\mathrm{f}}(W, S)$ (or simply $S^{f}$ ), by putting

$$
\mathrm{S}^{\mathrm{f}}=\left\{T \mid T \subset S \text { and } W_{T} \text { is finite }\right\} .
$$

This poset is partially ordered by inclusion. It is clear that $S^{f}-\{\emptyset\}$ is isomorphic to the poset of simplices of an abstract simplicial complex, which is denoted by $N(W, S)$ (or simply $N)$. We call $N$ the nerve of $(W, S)$.

TheOREM 2.6 (Davis, Moussong [7], [18]). Associated to a Coxeter system $(W, S)$, there is a PE cell complex $\Sigma(W, S)(=: \Sigma)$ with the following properties:

(1) The poset of cells in $\Sigma$ is the poset of cosets

$$
W \mathrm{~S}^{\mathrm{f}}=\coprod_{T \in \mathrm{S}^{\mathrm{f}}} W / W_{T} .
$$

(2) $W$ acts by isometries on $\Sigma$ with finite stabilizers and with compact quotient. 
(3) Each cell in $\Sigma$ is simple (so that the link of each vertex is a simplicial cell complex). In fact, this complex is just $N(W, S)$.

(4) $\Sigma$ is $\mathrm{CAT}(0)$.

3. Root system and essential elements of a Coxeter group. Recall from the introduction that for a Coxeter system $(W, S)$, there is a symmetric bilinear form (, ) on a real vector space $V$, having a basis $\left\{\alpha_{s} \mid s \in S\right\}$ in one-to-one correspondence with $S$.

Now, for each $s \in S$, define a linear transformation $\sigma_{s}: V \rightarrow V$ by $\sigma_{s} \lambda=\lambda-2\left(\alpha_{s}, \lambda\right) \alpha_{s}$. Then $\sigma_{s}$ is a linear reflection. It has order 2 and fixes the hyperplane $H_{s}=\left\{\delta \in V \mid\left(\delta, \alpha_{s}\right)=0\right\}$ pointwise, and $\sigma_{s} \alpha_{s}=-\alpha_{s}$. We have the following theorem (see [11], [16]).

TheOREM 3.1. There is a unique homomorphism $\sigma: W \rightarrow \mathrm{GL}(V)$ sending $s$ to $\sigma_{s}$. This homomorphism is a faithful representation of $W$ and the group $\sigma(W)$ preserves the bilinear form. Moreover, for each pair $s, t \in S$, the order of st in $W$ is precisely $m_{s t}$.

From now on, we write $w(\alpha)$ for $\sigma(w)(\alpha)$, when $\alpha \in V$ and $w \in W$.

The root system $\Phi$ of $W$ is defined to be the collection of all vectors $w\left(\alpha_{s}\right)$, where $w \in W$ and $s \in S$. An important fact about the root system is that any root $\alpha \in \Phi$ can be expressed as

$$
\alpha=\sum_{s \in S} c_{s} \alpha_{s},
$$

where all the coefficients satisfy $c_{s} \geq 0$ (we call $\alpha$ a positive root and write $\alpha>0$ ), or all the coefficients satisfy $c_{s} \leq 0$ (call $\alpha$ a negative root and write $\alpha<0)$. Write $\Phi^{+}$and $\Phi^{-}$for the respective sets of positive and negative roots. Then $\Phi^{+} \cap \Phi^{-}=\emptyset, \Phi^{+} \cup \Phi^{-}=\Phi$ and $\Phi^{-}=-\Phi^{+}$. The map from $\Phi$ to $R=\left\{w t w^{-1} \mid w \in W, t \in S\right\}$ (the set of reflections in $W$ ) given by $\alpha=w\left(\alpha_{s}\right) \mapsto w s w^{-1}$ is well defined and restricts to a bijection from $\Phi^{+}$ (and $\Phi^{-}$) to $R$, and $\sigma\left(w s w^{-1}\right)=t_{\alpha}$, where $t_{\alpha}$ is the linear reflection given by $t_{\alpha} \lambda=\lambda-2(\alpha, \lambda) \alpha$. The following fact is important when discussing root systems.

Proposition $3.2([11],[16])$. Let $w \in W, \alpha \in \Phi^{+}$. Then $l\left(w t_{\alpha}\right)>l(w)$ if and only if $w(\alpha)>0$.

The statements in the remaining part of this section are mostly due to Krammer [17], as revised by Paris [19]. Let $u, v \in W$ and $\alpha \in \Phi$. We say that $\alpha$ separates $u$ and $v$ if $u \alpha \in \Phi^{\varepsilon}$ and $v \alpha \in \Phi^{-\varepsilon}$, where $\varepsilon \in\{+,-\}$. Let $w \in W$ and $\alpha \in \Phi$. We say that $\alpha$ is $w$-periodic if there is some positive integer $m$ such that $w^{m} \alpha=\alpha$.

Lemma 3.3 ([19]). Let $w \in W$ and $\alpha \in \Phi$. Then exactly one of the following holds: 
(1) $\alpha$ is w-periodic.

(2) $\alpha$ is not $w$-periodic, and the set $\left\{m \in \mathbb{Z} \mid \alpha\right.$ separates $w^{m}$ and $\left.w^{m+1}\right\}$ is finite and has an even cardinality.

(3) $\alpha$ is not $w$-periodic, and the set $\left\{m \in \mathbb{Z} \mid \alpha\right.$ separates $w^{m}$ and $\left.w^{m+1}\right\}$ is finite and has an odd cardinality.

We say that $\alpha$ is $w$-even in case (2), and $w$-odd in case (3).

Lemma $3.4([19])$. Let $\alpha \in \Phi, w \in W$, and $p \in \mathbb{N}, p \geq 1$. Then:

(1) $\alpha$ is $w$-periodic if and only if $\alpha$ is $w^{p}$-periodic.

(2) $\alpha$ is w-even (resp., w-odd) if and only if $\alpha$ is $w^{p}$-even (resp., w-odd).

A subgroup $G$ is called a parabolic subgroup of a Coxeter group $(W, S)$ if $G=x W_{I} x^{-1}$ for some $x \in W$ and $I \subset S$. An element $w \in W$ is called essential if it does not lie in any proper parabolic subgroup of $W$.

Following Krammer [17], we define the parabolic closure $\operatorname{Pc}(A)$ of a subset $A$ of a Coxeter group $W$ to be the intersection of all parabolic subgroups containing $A$. In [20], the present author proves that $\operatorname{Pc}(A)$ is a parabolic subgroup of $W$. With this terminology, an element $w \in W$ is essential if and only if $\operatorname{Pc}(w)=W$.

Paris shows in [19] the existence of essential elements.

Proposition 3.5 (Paris [19]). If $(W, S)$ is a Coxeter group, where $S=$ $\left\{s_{1}, \ldots, s_{n}\right\}$, then $c=s_{n} \cdots s_{1}$ (called a Coxeter element) is an essential element of $W$.

In [19] Paris attributes the following result to Krammer [17].

TheOREM 3.6. For an irreducible, infinite Coxeter group $(W, S)$, an element $w \in W$ is essential if and only if $W$ is generated by the set $\left\{t_{\alpha} \mid \alpha \in \Phi^{+}\right.$ and $\alpha$ is $w$-odd\}.

To the author's understanding, this statement does not appear in Krammer's thesis [17], but it can be proved using some results that Krammer has established. A proof of Theorem 3.6 is included at the end of this section for the sake of completeness.

The next result follows from Theorem 3.6 and the discussion in the preceding paragraphs.

Corollary 3.7 ([19]). Assume that $W$ is an irreducible, infinite Coxeter group. Let $w \in W$ and $p$ be a positive integer. Then $w$ is essential if and only if $w^{p}$ is essential.

The following theorem appears in Krammer [17, p. 69].

THEOREM 3.8. Assume that $W$ is an irreducible, infinite, nonaffine Coxeter group. Let $w \in W$ be an essential element. Then $\langle w\rangle=\left\{w^{m} \mid m \in \mathbb{Z}\right\}$ is a finite index subgroup of the centralizer $C(w)$ of $w$ in $W$. 
Corollary 3.7 and Theorem 3.8 play an important role in the proofs of the theorems stated in the introduction.

To prove Theorem 3.6, we need some results from Krammer [17].

A $w$-periodic root $\alpha$ is called $w$-critical $([17$, p. 53]) if it satisfies the condition: The bilinear form (defined in the introduction) is positive definite on $\operatorname{Span}\{\langle w\rangle \alpha\}$ and $\sum_{n=1}^{k} w^{n} \alpha=0$, where $k$ is the smallest positive integer with $w^{k} \alpha=\alpha$.

Lemma 3.9 ([17, p. 54]). The subgroup generated by $\left\{t_{\alpha} \mid \alpha\right.$ is w-critical $\}$ is a finite subgroup of $W$.

TheOREM 3.10 ([17, p. 56]). The parabolic closure $\operatorname{Pc}(w)$ of $w$ equals the subgroup of $W$ generated by the reflections $\left\{t_{\alpha} \mid \alpha\right.$ is w-critical or $w$-odd $\}$.

Let $W_{1}$ be the subgroup generated by $\left\{t_{\alpha} \mid \alpha\right.$ is $w$-odd $\}$ and $\Phi_{1}=$ $\left\{w_{1}(\alpha) \mid w_{1} \in W_{1}, \alpha\right.$ is $w$-odd $\}$.

Lemma 3.11 ([17, p. 56]). Let $\alpha$ be w-periodic. Then either $(\alpha, \beta)=0$ for any $\beta \in \Phi_{1}$, or $\alpha \in \Phi_{1}$.

Lemma 3.12 ([16, p. 131]). Assume that $(W, S)$ is irreducible. Then any proper $W$-invariant subspace is contained in the radical $V^{\perp}$ (of the bilinear form $)$, i.e., $V^{\perp}=\left\{v \in V \mid\left(v, \alpha_{s}\right)=0\right.$ for any $\left.s \in S\right\}$.

Proof of Theorem 3.6. Proposition 3.5 states that essential elements exist in a Coxeter group. Now, assume that $(W, S)$ is an irreducible, infinite Coxeter group. For any essential element $w$ of $W, \operatorname{Pc}(w)=W$. It follows from Lemma 3.9 and Theorem 3.10 that $\Phi_{1}$ is nonempty. Let $V_{0}$ be the subspace spanned by those $w$-critical roots $\alpha$ satisfying the condition $(\alpha, \beta)=0$ for any $\beta \in \Phi_{1}$. Then Theorem 3.10 and Lemma 3.11 imply that $V_{0}$ is a $W$-invariant subspace. Obviously, $V_{0} \neq V$, since otherwise $(\beta, \beta)=0$ for $\beta \in \Phi_{1}$, which is absurd. It follows from Lemma 3.12 that $V_{0} \subset V^{\perp}$. This is impossible unless $V_{0}=0$, since any critical root $\alpha \in V_{0}$ satisfies $(\alpha, \alpha)=1$. Therefore, if $w \in W$ is an essential element, $W=\operatorname{Pc}(w)$ is generated by $w$-odd reflections. The converse is clear from Theorem 3.10.

4. Proofs of the theorems. Now we come to the proofs of the theorems stated in the introduction. We begin with a lemma.

LEMma 4.1. If an irreducible Coxeter group $(W, S)$ contains an infinite cyclic subgroup $\langle x\rangle$ as a finite index subgroup, then $W \cong D_{\infty}$, the infinite dihedral group.

Proof. The key point is that $W$ acts on the $\operatorname{CAT}(0)$ space $\Sigma=\Sigma(W, S)$ properly and cocompactly. What follows is similar to the proof of the Flat Torus Theorem for CAT(0) spaces (see page 246 in [3]). Since $[W:\langle x\rangle]$ is finite, there is a positive integer $k$ such that $\left\langle x^{k}\right\rangle$ is a normal subgroup. It is 
a finite index subgroup of $W$. The set $\operatorname{Min}\left(\left\langle x^{k}\right\rangle\right)$ of points in $\Sigma$ which are moved minimal distance by $x^{k}$ is a nonempty closed subspace of $\Sigma$ isometric to one of the form $Y \times \mathbb{R}$, where $Y$ is a closed convex subspace of $\Sigma$ and $\mathbb{R}$ is the set of real numbers. (For any group $\Gamma$ acting on a CAT(0) space by isometries, $\operatorname{Min}(\Gamma)$ is a subspace as defined on page 229 in [3].)

Now, $x^{k}$ acts as a nontrivial translation on the factor $\mathbb{R}$ and acts as an identity map on $Y$. Since $\left\langle x^{k}\right\rangle \subset W$ is normal, $W$ acts by isometries of $\operatorname{Min}\left(\left\langle x^{k}\right\rangle\right)$, preserving the splitting. By properties of $\mathrm{CAT}(0)$ spaces, the fixed point set $Y_{1}$ of the induced action of the finite group $W /\left\langle x^{k}\right\rangle$ on the complete $\mathrm{CAT}(0)$ space $Y$ is a nonempty, closed, convex subset of $Y$. By construction $Y_{1} \times \mathbb{R}$ is $W$-invariant and the action of $W$ on the factor $Y_{1}$ is the identity.

Pick $y \in Y_{1}$ and consider the action of $W$ on $L=\{y\} \times \mathbb{R}$. The restriction of each $s$ to $L$ is either a reflection or the identity. Since $x^{k}$ is a translation on $L$, there are elements $s_{1}$ and $s_{2}$ in $S$ which act as different reflections on $L$. So, the order of $s_{1} s_{2}$ is infinite. By considering the collection of right cosets $\left\{\left\langle x^{k}\right\rangle\left(s_{1} s_{2}\right)^{l} \mid l \in \mathbb{Z}\right\} \subset\left\langle x^{k}\right\rangle \backslash W$, we know there is a positive integer $d$ such that $\left(s_{1} s_{2}\right)^{d} \in\left\langle x^{k}\right\rangle$. Hence, $\left[W:\left\langle s_{1} s_{2}\right\rangle\right]$ is finite, and therefore, so is $\left[W: W_{\left\{s_{1}, s_{2}\right\}}\right]$. This is impossible unless $W=W_{\left\{s_{1}, s_{2}\right\}}=D_{\infty}$, because of the following result proved by Deodhar [11]: For an irreducible, infinite Coxeter group $(W, S)$ and any proper subset $J$ of $S,\left[W: W_{J}\right]$ is infinite. (This result was rediscovered by T. Hosaka [14] using different methods.)

Proof of Theorem 1.1. Assume that $(W, S)$ is an irreducible, infinite, nonaffine Coxeter group and $G$ is a finite index subgroup of $W$. Let $Z(G)$ be the center of $G$. The proof is divided into two cases.

Case 1: $Z(G)$ contains an element $x$ of infinite order. By Proposition 3.5, we can pick an essential element $w$ of $W$. Since the number of cosets $\left\{G w^{m} \mid m \in \mathbb{Z}\right\}$ is finite, there is a positive integer $p$ such that $w^{p} \in G$. By Corollary 3.7, $w^{p}$ is essential in $W$. Since $x$ is in the centralizer $C\left(w^{p}\right)$ of $w^{p}$, the number of cosets $\left\{\left\langle w^{p}\right\rangle x^{m} \mid m \in \mathbb{Z}\right\} \subset\left\langle w^{p}\right\rangle \backslash C\left(w^{p}\right)$ is finite by Theorem 3.8 so there is a positive integer $q$ such that $x^{q} \in\left\langle w^{p}\right\rangle$. Now, $x^{q}$, and hence $x$, is essential in $W$. Since $G$ is contained in the centralizer $C(x)$ of $x$, it follows that the index $[G:\langle x\rangle] \leq[C(x):\langle x\rangle]$ is finite by Theorem 3.8. Therefore, $[W:\langle x\rangle]$ is finite. Now, Lemma 4.1 implies that $W$ is the infinite dihedral group $D_{\infty}$, which is impossible since $D_{\infty}$ is affine.

CASE 2: $Z(G)$ is a torsion subgroup, i.e., every element in $Z(G)$ has a finite order. A preliminary result used in the proof of the Solvable Subgroup Theorem for CAT(0) spaces (see [3, p. 247]) states that if a group $\Gamma$ acts properly and cocompactly by isometries on a $\mathrm{CAT}(0)$ space, then every abelian subgroup of $\Gamma$ is finitely generated. It follows that $Z(G)$ is finitely generated, and hence finite. A result of Tits states that any finite subgroup 
of a Coxeter group is contained in a finite parabolic subgroup. Therefore, in this case, the parabolic closure $\operatorname{Pc}(Z(G))$ of $Z(G)$ is a finite parabolic subgroup of $W$. Without loss of generality, we may assume that $\operatorname{Pc}(Z(G))$ is a finite special parabolic subgroup $W_{K}$, where $K \subset S$.

Since $Z(G)$ is normal in $G, g W_{K} g^{-1}$ is a parabolic subgroup containing $Z(G)$ for any $g \in G$. By the uniqueness of the parabolic closure (or by the discussion of the rank of the intersection of two parabolic subgroups in [20]), we have $g W_{K} g^{-1}=W_{K}$, and hence $G \subset N\left(W_{K}\right)$ (the normalizer of $W_{K}$ in $\left.W\right)$. Therefore, $\left[W: N\left(W_{K}\right)\right]$ is finite. This implies that the set $R_{1}=\left\{w t w^{-1} \mid t \in K, w \in W\right\}$ is finite.

Now, consider the reflection subgroup $W_{1}$ of $W$ generated by $R_{1}$. The group $W_{1}$ is a Coxeter group by [12] or [13], with a set $S_{1}$ of distinguished generators, where $S_{1} \subset \bigcup_{w_{1} \in W_{1}} w_{1} R_{1} w_{1}^{-1}$. (It is clear that $\bigcup_{w_{1} \in W_{1}} w_{1} R_{1} w_{1}^{-1}=R_{1}$ in the present situation.) Hence, the set of reflections in $W_{1}$, which by definition is $\left\{w_{1} t_{1} w_{1}^{-1} \mid t_{1} \in S_{1}, w_{1} \in W_{1}\right\}$ $\left(\subset R_{1}\right)$, is finite. Therefore, $W_{1}$ is a finite Coxeter group. Suppose that $\operatorname{Pc}\left(W_{1}\right)=y W_{L} y^{-1}$, where $y \in W$ and $L$ is a proper subset of $S$. Since $W_{1}$ is normal in $W$, we conclude that $y W_{L} y^{-1}$ is a (proper) normal subgroup of $W$. This is impossible, due to the result proved by Paris [19]: any proper nontrivial special subgroup of an irreducible Coxeter group is not normal. In the current situation, we need to replace the distinguished set $S$ of generators by $y S y^{-1}$.

In conclusion, $Z(G)=\{1\}$. This finishes the proof of Theorem 1.1.

Remark. Paris obtains the above-mentioned result based on R. Howlett's description of the normalizer of a special subgroup of a Coxeter group in [15], where Howlett discusses the situation of finite Coxeter groups. In fact, Howlett's description is valid in general. The proof is just a slight modification of that for finite Coxeter groups.

The proof of Theorem 1.2 is similar.

Proof of Theorem 1.2. Let $G$ be a finite index subgroup of an irreducible, infinite, nonaffine Coxeter group. Suppose that $G=A \times B$, where $A$ and $B$ are nontrivial subgroups. Pick an essential element $w$ in $W$. Following the idea in the proof of Theorem 1.1, we deduce that there is a positive integer $p$ such that $w^{p} \in G$. Now $w^{p}$ is essential and $w^{p}=a b$ for some $a \in A$ and $b \in B$. At least one of them, say $a$, has an infinite order, because $w^{p}$ has an infinite order, and $a$ and $b$ commute. Notice that $a$ is in the centralizer $C\left(w^{p}\right)$ of $w^{p}$, since $a w^{p}=w^{p} a$. By considering the collection of cosets $\left\{\left\langle w^{p}\right\rangle a^{m} \mid m \in \mathbb{Z}\right\}$, we conclude that there is a positive integer $q$ such that $a^{q} \in\left\langle w^{p}\right\rangle$. So, $a$ is an essential element. Since each element of $B$ commutes with $a$, it follows that $B \subset C(a)$, the centralizer of $a$. Since the 
collection of cosets $\{\langle a\rangle h \mid h \in B\}$ is finite and $A \cap B=\{1\}$, we know that $B$ is finite. Without loss of generality, we can assume that $\operatorname{Pc}(B)=W_{I}$, a finite parabolic subgroup. Since $B$ is normal in $G$, the uniqueness of parabolic closure implies that $G \subset N\left(W_{I}\right)$. So, $\left[W: N\left(W_{I}\right)\right]$ is finite. The arguments in Case 2 of the proof of Theorem 1.1 now apply to yield a contradiction. Hence, the conclusion of Theorem 1.2 holds.

Proof of Theorem 1.3. Assume that

$$
G=W_{1} \times \cdots \times W_{n} \quad \text { (internal direct product), }
$$

where each $W_{i}$ is an irreducible, infinite, nonaffine Coxeter group. Let $H$ be a finite index subgroup of $G$. Denote by $p_{i}: G \rightarrow W_{i}$ the projection of $G$ to the $i$ th factor $W_{i}$. Let $c=\prod_{i=1}^{n} c_{i} \in Z(H)$, the center of $H$, where $c_{i} \in p_{i}(H) \subset W_{i}$. Pick an arbitrary $h=\prod_{i=1}^{n} h_{i} \in H$ with $h_{i} \in p_{i}(H)$. Then $c h=h c$. This implies that $\prod_{i=1}^{n}\left(c_{i} h_{i}\right)=\prod_{i=1}^{n}\left(h_{i} c_{i}\right)$, so $c_{i} h_{i}=h_{i} c_{i}$ for any $i$. Notice that since $h_{i} \in p_{i}(H)$ is arbitrary, $c_{i} \in Z\left(p_{i}(H)\right)$. Since $W_{i}=p_{i}(G)$ and $\left[p_{i}(G): p_{i}(H)\right]=\left[G: p_{i}^{-1}\left(p_{i}(H)\right)\right] \leq[G: H]<\infty$, Theorem 1.1 implies that $c_{i}=1$. Hence, $Z(H)=\{1\}$.

Now we use induction on $n$, the number of factors in expression (4.1), to prove that if a finite index subgroup $H$ of $G$ can be expressed as

$$
H=H_{1} \times \cdots \times H_{m} \quad \text { (internal direct product), }
$$

where each $H_{i}$ is a nontrivial subgroup, then $m \leq n$.

The case $n=1$ is just Theorem 1.2. Now, assume $n \geq 2$ and $m \geq 2$. Notice that $p_{1}(H)=p_{1}\left(H_{1}\right) \cdots p_{1}\left(H_{m}\right)$, and

$$
p_{1}\left(H_{i}\right) \cap\left\{p_{1}\left(H_{1}\right) \cdots p_{1}\left(H_{i-1}\right) p_{1}\left(H_{i+1}\right) \cdots p_{1}\left(H_{m}\right)\right\}=\{1\},
$$

because this intersection is contained in $Z\left(p_{1}(H)\right)$, which is trivial by Theorem 1.1 (knowing that $p_{1}(H)$ is a finite index subgroup of $W_{1}$ ). Hence

$$
p_{1}(H)=p_{1}\left(H_{1}\right) \times \cdots \times p_{1}\left(H_{m}\right) .
$$

By Theorem 1.2 only one of the factors on the right-hand side, say $p_{1}\left(H_{1}\right)$, can be nontrivial, and all other $p_{1}\left(H_{j}\right)(j \neq 1)$ are trivial. So, $p_{1}\left(H_{1}\right)=$ $p_{1}(H)$. Without loss of generality, we can assume that $p_{i}\left(H_{1}\right)$ is nontrivial for $i=1, \ldots, l$, and is trivial for $i=l+1, \ldots, m$. This implies that $p_{i}\left(H_{j}\right)=\{1\}$ for $i=1, \ldots, l, j \neq 1$. Hence,

$$
H_{1} \subset W_{1} \times \cdots \times W_{l}, \quad H_{2} \times \cdots \times H_{m} \subset W_{l+1} \times \cdots \times W_{n} .
$$

Now use the induction hypothesis and the following simple fact (the proof of which is left to the reader):

Lemma 4.2. Let $G_{1}$ and $G_{2}$ be two groups. If $N_{i}$ is a subgroup of $G_{i}$, $i=1,2$, and $\left[G_{1} \times G_{2}: N_{1} \times N_{2}\right]<\infty$, then $\left[G_{i}: N_{i}\right]<\infty, i=1,2$, and $\left[G_{1} \times G_{2}: N_{1} \times N_{2}\right]=\left[G_{1}: N_{1}\right]\left[G_{2}: N_{2}\right]$. 
We conclude that $m \leq n$.

Having this inequality in mind, we may continue to decompose some factors in expression (4.2), until no factor can be further decomposed (it is a finite step procedure due to the above inequality). From now on, when we talk about a decomposition of the form (4.2), we assume that no factor can be further decomposed.

Suppose that there is another decomposition

$$
H=K_{1} \times \cdots \times K_{r},
$$

where no factor $K_{j}$ can be further decomposed. Let $q_{j}: H \rightarrow K_{j}$ be the projection of $H$ onto its $j$ th factor in the decomposition (4.3). We know that $K_{i}=q_{i}(H)=q_{i}\left(H_{1}\right) \cdots q_{i}\left(H_{m}\right)$. And notice that $q_{i}\left(H_{k}\right) \cap \prod_{j \neq k} q_{i}\left(H_{j}\right)$ $=\{1\}$, because this intersection is contained in the center $Z\left(K_{i}\right)$ of $K_{i}$, and $Z\left(K_{i}\right) \subset Z(H)$, while the latter is trivial by the first part of this theorem. We thus have

$$
K_{i}=q_{i}\left(H_{1}\right) \times \cdots \times q_{i}\left(H_{m}\right) .
$$

By the assumption that $K_{i}$ cannot be further decomposed, $K_{i}=q_{i}\left(H_{\phi(i)}\right)$ for some $\phi(i)$, and $q_{i}\left(H_{j}\right)$ is trivial for $j \neq \phi(i)$. So, $\phi$ defines a map from $\{1, \ldots, r\}$ to $\{1, \ldots, m\}$, and it is surjective because, for any $j \in\{1, \ldots, m\}$, there is an $i \in\{1, \ldots, r\}$ such that the restriction $\left.q_{i}\right|_{H_{j}}$ is nontrivial. Hence $m \leq r$. Similar arguments also yield $r \leq m$. Therefore, $m=r$ and $\phi$ is a bijection. After re-indexing, we may assume $\phi=$ id (the identity map). This means that $H_{i} \subset K_{i}$ and the restriction $\left.q_{i}\right|_{H_{i}}$ is indeed the inclusion $H_{i} \hookrightarrow K_{i}$. Since

$$
H=H_{1} \times \cdots \times H_{m}=K_{1} \times \cdots \times K_{m},
$$

we know that $H_{i}=K_{i}$ for $i=1, \ldots, m$. This is the claimed unique decomposition of $H$ and $m$ is uniquely determined by $H$. The proof of Theorem 1.3 is complete.

The situation that $m<n$ may happen. To illustrate this, we need the following lemma.

Lemma 4.3. Let $W=W_{1} \times W_{2}$, where $W_{1}$ and $W_{2}$ are irreducible, infinite, nonaffine Coxeter groups. If $H$ is a finite index subgroup of $W$ and $H=H_{1} \times H_{2}$, where $H_{i} \neq\{1\}, i=1,2$, then after re-indexing, $H_{i} \subset W_{i}$.

Proof. As in the proof of Theorem 1.3, let $p_{i}: W \rightarrow W_{i}$ be the projection. It follows that $p_{1}(H)$ is a finite index subgroup of $W_{1}$ and $p_{1}(H)=$ $p_{1}\left(H_{1}\right) \times p_{1}\left(H_{2}\right)$ by repeating the arguments in the proof of Theorem 1.3. In this product decomposition, only one factor, say $p_{1}\left(H_{1}\right)$, is nontrivial by Theorem 1.2. So, $p_{1}(H)=p_{1}\left(H_{1}\right)$ and $p_{1}\left(H_{2}\right)=\{1\}$. This implies that $H_{2} \subset W_{2}$. So, $p_{2}\left(H_{2}\right) \neq\{1\}$. By Theorem $1.2, p_{2}\left(H_{1}\right)=\{1\}, H_{1} \subset W_{1}$. 
In the following two examples, let $\left(W_{i}, S_{i}\right)$ be an irreducible, infinite, nonaffine Coxeter group, $i=1,2$. Suppose that $W=W_{1} \times W_{2}$ and $S=$ $S_{1} \cup S_{2}$.

Example 4.4 (M. Davis). Suppose that $f_{i}: W_{i} \rightarrow G(i=1,2)$ is a surjective homomorphism from $W_{i}$ to a finite group $G$. Let $H=\left\{\left(w_{1}, w_{2}\right) \in W \mid\right.$ $\left.f_{1}\left(w_{1}\right)=f_{2}\left(w_{2}\right)\right\}$. Then $[W: H]=|G|, p_{1}(H)=W_{1}$ and $p_{2}(H)=W_{2}$, where $p_{i}$ is the projection defined in Lemma 4.3. It follows from Lemma 4.3 that $H$ cannot be further decomposed.

EXAMPLE 4.5. Let $\phi: W \rightarrow D_{1}$ be the surjective homomorphism such that $\phi(s)=-1$ for any $s \in S$, where $D_{1}=\{-1,1\}$. Let $H=\operatorname{ker}(\phi)$. Then $[W: H]=2$. If $H$ had a decomposition $H=H_{1} \times H_{2}, H_{i} \neq\{1\}$, then one of the factors, say $H_{1}$, would be $W_{1}$ because of Lemmas 4.3 and 4.2 and $[W: H]=2$. This would imply that $\phi\left(s_{1}\right)=1$ for any $s_{1} \in S_{1}$, a contradiction. Hence, $H=\operatorname{ker}(\phi)$ cannot be further decomposed.

Proof of Theorem 1.4. Let $w \neq 1$ be an element of $W$. We want to prove that $[W: C(w)]=\infty$. The proof is divided into two cases.

CASE 1: The order of $w$ is finite. In this case, the parabolic closure $\operatorname{Pc}(\langle w\rangle)$ of $\langle w\rangle$ is a finite parabolic subgroup. Without loss of generality, assume that $\operatorname{Pc}(\langle w\rangle)=W_{K}$, where $K \subset S$. The uniqueness of the parabolic closure and the fact that $g w g^{-1}=w$ for any $g \in C(w)$ imply that $g W_{K} g^{-1}=W_{K}$ for $g \in C(w)$. Hence, $C(w) \subset N\left(W_{K}\right)$. The discussion of Case 2 in the proof of Theorem 1.1 yields $\left[W: N\left(W_{K}\right)\right]=\infty$ when $W$ is an irreducible, infinite Coxeter group and $W_{K}$ is a nontrivial finite special subgroup. Therefore, in this situation, $[W: C(w)]=\infty$.

CASE 2: The order of $w$ is infinite. Suppose that $[W: C(w)]<\infty$. Pick an essential element $x \in W$. Since the number of cosets $\left\{C(w) x^{k} \mid k \in \mathbb{Z}\right\}$ is finite, there is a positive integer $m$ such that $x^{m} \in C(w)$. By Corollary 3.7, $x^{m}$ is essential. Notice that $w \in C\left(x^{m}\right)$ and the number of cosets $\left\{\left\langle x^{m}\right\rangle w^{l} \mid\right.$ $l \in \mathbb{Z}\}$ in $C\left(x^{m}\right)$ is finite because of Theorem 3.8. We conclude that there is a positive integer $n$ such that $w^{n} \in\left\langle x^{m}\right\rangle$. Now, Corollary 3.7 implies that $w^{n}$ is essential, and hence, so is $w$. Then, by Theorem 3.8, we have $[W:\langle w\rangle]<\infty$. It follows from Lemma 4.1 that $W=D_{\infty}$, contradicting the assumption that $W$ is nonaffine.

In conclusion, $[W: C(w)]=\infty$ for $w \neq 1$. The assertion of the theorem follows immediately.

Acknowledgements. The author would like to thank Michael Davis and Tadeusz Januszkiewicz for the inspiration and numerous discussions. The author would also like to thank the referee for helpful suggestions. 


\section{References}

[1] Y. Benoist et P. de la Harpe, Adhérence de Zariski des groupes de Coxeter, Compos. Math. 140 (2004), 1357-1366.

[2] N. Bourbaki, Lie Groups and Lie Algebras, Chapters 4-6, Springer, New York, 2002.

[3] M. Bridson and A. Haefliger, Metric Spaces of Non-Positive Curvature, Springer, Berlin, 1999.

[4] K. S. Brown, Buildings, Springer, New York, 1989.

[5] Y. de Cornulier et P. de la Harpe, Décompositions de groupes par produit direct et groupes de Coxeter, arXiv:math.GR/0507366.

[6] M. Davis, Groups generated by reflections and aspherical manifolds not covered by Euclidean space, Ann. of Math. (2) 117 (1983), 293-325.

[7] -, Nonpositive curvature and reflection groups, in: Handbook of Geometric Topology, R. J. Daverman and R. B. Sher (eds.), Elsevier, Amsterdam, 2002, 373-422.

[8] - The cohomology of a Coxeter group with group ring coefficients, Duke Math. J. 91 (1998), 297-314.

[9] —, Geometry and topology of Coxeter groups, http://www.math.ohio-state.edu/ mdavis/davisbook.pdf.

[10] M. Davis, J. Dymara, T. Januszkiewicz and B. Okun, Weighted $L^{2}$-cohomology of Coxeter groups, arXiv:math.GT/0402377.

[11] V. V. Deodhar, On the root system of a Coxeter group, Comm. Algebra 10 (1982), 611-630.

[12] - A note on subgroups generated by reflections in Coxeter groups, Arch. Math. (Basel) 53 (1989), 543-546.

[13] M. Dyer, Reflection subgroups of Coxeter systems, J. Algebra 135 (1990), 57-73.

[14] T. Hosaka, Parabolic subgroups of finite index in Coxeter groups, J. Pure Appl. Algebra 169 (2002), 215-227.

[15] R. Howlett, Normalizers of parabolic subgroups of reflection groups, J. London Math. Soc. 21 (1980), 62-80.

[16] J. E. Humphreys, Reflection Groups and Coxeter Groups, Cambridge Univ. Press, Cambridge, 1990.

[17] D. Krammer, The conjugacy problem for Coxeter groups, Ph.D. thesis, Universiteit Utrecht, 1994.

[18] G. Moussong, Hyperbolic Coxeter groups, Ph.D. thesis, The Ohio State Univ., Columbus, OH, 1988.

[19] L. Paris, Irreducible Coxeter groups, arXiv:math.GR/0412214, v1 and v2.

[20] D. Qi, A note on parabolic subgroups of a Coxeter group, arXiv:math.GR/0512408.

[21] L. Solomon, A Mackey formula in the group ring of a Coxeter group, J. Algebra 41 (1976), 255-268.

[22] J. Tits, Buildings of Spherical Type and Finite BN-pairs, Lecture Notes in Math. 386, Springer, Berlin, 1974.

Department of Mathematics

The Ohio State University

231 West 18th Avenue

Columbus, OH 43210, U.S.A.

E-mail:dwqi@math.ohio-state.edu

Received 28 March 2006;

in revised form 10 July 2006 